\section{Manchester Literary and Philosophical Society}

Mr. L. Cohen's presidential address, "A Shopkeeper's Notes on his Trade", has been published in a recent issue of the Memoirs and Proceedings of the Manchester Literary and Philosophical Society $(106,1963-64 . \quad$ Pp. $131+$ lii +4 plates. Manchester: Manchester Literary and Philosophical Society, 1964. 31s. 6d.). This issue also contains Mr. M. J. B. Birks's account of the geography, recent history, peat stratigraphy and pollen analysis of Chat Moss; Prof. D. G. Christopherson's comparison of university practice in Great Britain and the United States; Prof. H. J. Eysenck's review of the causes and cures of neuroses; A. P. Hatton and J. W. Flowett's account of Clegg's model of a Watt ocean engine; a brief assessment of the future of atomic power by S. A. Ghalib; and an account by R. Frankenberg of John Aiken (17471822)-doctor and philosopher.

\section{Maxwell's International Subject Bibliographies}

Maxwell's International Subject Bibliographies, Special Subject Bibliography, No. 13 (second edition, July 1964), covers textiles, fashion, elothing, dressmaking and associated subjects for the period 1961-June 1964. No. 16 (second edition, September 1964) deals with child psychiatry and psychology, including psychopathology, psychodynamics and psychoanalysis of children, and covers the period 1953-June 1964. No. 20 (second edition) is concerned with vacuum science and technology and covers the period 1958-July 1964. No. 37 (second edition) deals with tropical agriculture (including soil science, forestry, sylviculture, horticulture, livestock, bee-keeping, fisheries) and covers the period 1960-September 1964.

\section{An Improved Means of Bleaching in Paper-making}

BLEACHING is a most important, if not a key stage, in the production of pulp used in manufacture of many typos of paper. This treatment aids purification of pulp fibres, such as wood and waste paper, and imparts to them the required degree of whiteness. There are numerous methods of bleaching pulp, mostly dependent on chemical oxidation in which chlorine derivatives are used in a multi-stage system; for example, in the case of bleaching powder (calcium chloro-hypochloride, otherwise 'chloride of lime'), the active agent is essentially nascent oxygen. An alternative oxidation system favoured in recent years involves the use of sodium and hydrogen peroxides. There is, however, another standard technique used in bleaching paper-pulp, one based on a powerful reducing agent such as zinc hyposulphite ('hydrosulphite'). A new, high-purity compound of this type is now being produced under the name 'Protolin $Z$ ', which is claimed by the manufacturers, Rohm and Haas Co., Philadelphia, as offering several advantages over other bleaching tech. niques; a brief account of this is given in Rohm and Haas Reporter (22, No. 5; September-October, 1964, Philadelphia, Pa. 19105; subsidiary Lennig Chemicals, Ltd., London, W.C.1). "Protolin $Z$. . provides a substantial improvement in pulp brightness at low cost. Moreover, bleaching may be carried out in existing or only slightly modified equipment. In contrast, bleaching with chlorine and peroxide usually calls for specialized equipment." The same Company also supplies another form of 'hydrosulphite' known as 'Lykopon', a highly purified sodium derivative. In bleaching of pulp both products are interchangeable in terms of performance, but 'Protolin $Z$ ' is generally preferred on the basis of lower cost. This latter compound provides substantial increase in 'brightness' in the sense of reflectance value as understood by the paper industry in measuring level of colour. A further attribute of 'Protolin $Z$ ' is that, as a highly uniform product, free-flowing, non-dusting, white powder with excellent stability, this new grade of zine hyposulphite bleach is ideally suited for automatic feeding devices in modern paper-making machinery.

\section{Modern Methods of Manufacturing Formalin}

Formalin, the commercial solution of formaldehyde, has long been known as a preservative for biological specimens. In this age of plasties, however, it has perhaps en even more important application; it is one of the basic raw materials used in making synthetic resins, especially for wood adhesives and textile finishes. The formalin production plants at CIBA (A.R.L.), Duxford, operate on a truly large scale; they, and the process involved, are described in the latest issue of CIBA Journal (No. 32, Winter 1964,65; CIBA, Ltd., Basle, Switzerland). At the present time there are four plants, the latest and largest having only recently been put into operation. These plants are of particular interest because they operate continuously and are almost completely automatic; their design enables them to be manned by only eight men working in four shifts. The process used is one of dehydrogenation and oxidation of methanol, passed with air over a crystalline silver catalyst, at about $600^{\circ} \mathrm{C}$, by means of which a solution of formaldehyde and methanol in water is produced. The final product is stated to be a 40 per cent $\mathrm{v} / \mathrm{v}$ (by volume) solution with $36 \cdot 6$ per cent $\mathrm{w} / \mathrm{w}$ (by weight) formaldehyde and 6.5 per cent $\mathrm{w} / \mathrm{w}$ methanol. Control instrumentation is applied throughout all plants; all four installations are controlled from one control room. Strict fire and safety precautions are maintained because of the inflammability of methanol and the wide explosive range of methanol-water-air mixtures. Another important factor in the process is purity of raw materials, particularly as a safeguard against catalyst poisoning; a continuous check is made of all liquids in contact with the catalyst, which is itself analysed both before and after use. "The product is analysed for specific gravity and for formaldehyde, methanol and formic acid content at regular intervals. The formalin supplied to users is manufactured to a predetermined specification." This article is not only fully explanatory of both plant and process, but it also includes impressive illustrations of two of these plants and instrument panels in the control room, and also a flow diagram providing a schematic view of the formalin manufacturing process as practised at Duxford.

\section{Persistence of Insecticides in Soil}

A GOOD deal of publicity has recently been given to public anxiety about pesticides generally, and insecticides in particular, in their transmission in food chains and possible harm to both human and wild life. The documented review of scientific investigations on the question by C. A. Edwards entitled, "Factors Affecting the Persistence of Insecticides in the Soil", which appears in Soils and Fertilizers is, therefore, welcome $(27$, No. 6, 451; 1964). Many insecticidal chemicals are now applied directly to the soil or reach the soil from foliar sprays, and the most common residues are chlorinated hydrocarbons, other insecticides breaking down quite rapidly. Numerous related factors are obviously concerned in the volatilization, degradation or leaching of these materials; it seems that proportionately more is lost in a given time from a small than from a large dose and the rate of disappearance at a particular dosage is fairly constant after the initial, more rapid, loss. The loss increases with rise in temperature unless the soil is dry, because both volatilization and microbial activity are increased by moisture and heat. The persistent insecticides are not easily dissolved but may be carried down a slope or into the soil mechanically and the bulk of the residues is usually found in the top few inches, even after cultivation. The insecticide is held by the organic matter in the soil so that it persists much longer in a humose than in a mineral soil. On average, the percentages of material applied remaining after one year are about 80 for DDT, 75 for dieldrin, 60 for lindane, 55 for chlordane, 45 for heptachlor and 26 for aldrin, and the half-life of dieldrin may be $4-7$ years. 